\title{
REMISSION OF A CASE OF MULTIPLE HYMENOPTERA STINGS-ASSOCIATED CHRONIC URTICARIA DURING VENOM IMMUNOTHERPY
}

\author{
Marco Dubini ${ }^{1}$, Valerio Pravettoni ${ }^{2}$, Federica Rivolta ${ }^{2}$, Giulia Segatto ${ }^{1}$, Riccardo Asero ${ }^{3}$, \\ and Nicola Montano ${ }^{4}$ \\ ${ }^{1}$ Allergy and Clinical Immunology Residency, University of Milan, Italy \\ ${ }^{2}$ U.O.C. General Medicine - Immunology and Allergology, Fondazione Ca' Granda IRCCS, \\ Ospedale Maggiore Policlinico, Università di Milano \\ ${ }^{3}$ Clinica San Carlo \\ ${ }^{4}$ Fondazione IRCCS Ca ${ }^{6}$ Granda - Ospedale Maggiore; University of Milan
}

January 20, 2021

\begin{abstract}
The role of Immunotherapy in Chronic Urticaria is unclear, except for isolated circumstances. Hymenoptera sting causes acute urticaria and no report of $\mathrm{CU}$ after Hymenoptera sting can be found in the literature. We describe a case of onset of CU after multiple wasp stings that remitted during venom immunotherapy.
\end{abstract}

TITLE: REMISSION OF A CASE OF MULTIPLE HYMENOPTERA STINGS-ASSOCIATED CHRONIC URTICARIA DURING VENOM IMMUNOTHERPY

AUTHORS: Dubini Marco ${ }^{1}$, Pravettoni Valerio ${ }^{2}$, Rivolta Federica ${ }^{2}$, Segatto Giulia ${ }^{1}$, Asero Riccardo ${ }^{3}$, Nicola Montano ${ }^{1}$

1 - Allergy and Clinical Immunology Residency,

2 - General Medicine, Immunology and Allergology Unit, IRCCS Foundation Ca' Granda, Ospedale Maggiore Policlinico, Milan, Italy

3 - Ambulatorio di Allergologia, Clinica San Carlo, Paderno Dugnano, Milan, Italy.

Corresponding Author: Marco Dubini, marco.dubini@unimi.it, via Pace 920122 Milan, Italy.

KEYWORDS : chronic urticaria; venom immunotherapy; immunotherapy; venom allergy; Hymenoptera venom allergy.

INTRODUCTION

Chronic Urticaria (CU) is defined as the appearance of wheals, angioedema or both for more than 6 weeks due to known or unknown causes. Urticaria affects about $15-20 \%$ of the population once or more during a lifetime and $0.5-1.0 \%$ suffer from CU. So far, IgE-mediated mechanisms and the relevance of external allergens in $\mathrm{CU}$ have not been fully clarified. ${ }^{1}$

CASE REPORT 
We report a case of 34-year-old man with CU that appeared after a grade I Mueller reaction to Hymenoptera sting and remitted during venom immunotherapy (VIT), after months of unsuccessful therapy for CU.

He had a negative history of allergic and autoimmune diseases, and previous anaphylactic episodes, only a familiar history of intolerance to Aspirin and a personal history of gastroesophageal reflux disease, not requiring any therapy at that moment. He referred the first sting at the age of 20 by an unidentified insect and experienced a normal local reaction (smaller than diameter). Afterwards, about one year before our examination, he was stung by a vespid, and again experienced a normal local reaction. Two months later, about 20 minutes after receiving multiple stings by a wasp swarm, itching hives developed all over his body. At the local hospital intravenous methylprednisolone acetate and intramuscular chlorphenamine were administered, with complete regression in 24 hours. The day after, though, he manifested satellite lymphadenitis, which regressed in 5 days with steroid cream. Three weeks later, migrant generalized urticarial lesions started, showing spontaneous short-lived wheals not in relation to foods or drugs, physical agents or exercise. At our facility, routine blood tests were normal, except for mild leucopenia, which was already known before urticaria occurred; basophil and eosinophil counts were normal. Other causes of CU, such as acute or chronic infections and thyroid gland disorders, were excluded. Plasma levels of D-Dimer and acute phase reactants were normal. Autologous serum skin test excluded the presence of functional autoantibodies. Skin tests with common inhalant and food allergens (, ), Apis mellifera (, ) and Polistes dominula () venoms were negative. Vespinae venoms tested positive, both Vespula spp (ALK -Abelló) and Vespa crabro(Anallergo), at concentration of $0.1 \mu \mathrm{g} / \mathrm{mL}$. Specific IgE tested positive for vespid venoms, respectively $5.46 \mathrm{kU} / \mathrm{L}$ for Vespula spp, $0.47 \mathrm{kU} / \mathrm{L}$ V. crabro, $0.69 \mathrm{kU} / \mathrm{L}$ P. dominula. Analysis of the venom-specific component revealed rVes v $5(6.17 \mathrm{kU} / \mathrm{L})$, rVes v $1(<0.10 \mathrm{kU} / \mathrm{L})$, and $\mathrm{rPol} \mathrm{d} 5(1.07 \mathrm{kU} / \mathrm{L})$. Total IgE level was $132 \mathrm{kU} / \mathrm{L}$ and serum baseline tryptase $7 \mathrm{mcg} / \mathrm{mL}$. Notably, after skin tests with vespid venoms the patient suffered from an exacerbation of urticaria on his hips and thighs. Baseline assessment of disease activity with the urticaria activity score (UAS) showed a scarce control with oral antihistamine. Then he started oral prednisone, reaching the daily lowest effective dose of $5 \mathrm{mg}$. After three months, CU symptoms partially improved, and he agreed to start VIT because of the impairment of his quality of life. Venom-specific immunotherapy was started with Vespula spp extract ( $A L K$-Abelló) according to a 6-week cluster schedule with weekly incremental doses of venom extract subcutaneously, until a maintenance dose of 100,000 standard quality units $(\mathrm{SQ}-\mathrm{U} / \mathrm{ml})$ was reached. During the up-dosing phase, the patient remained under antihistamines therapy, because urticaria was still present. The achieved maintenance dose $(100,000$ SQ-U/ml) was then given every 4-6 weeks. During the up-dosing phase and maintenance treatment minor side-effects such as local flushing and mild itching were reported and tolerated, and a worsening of the urticarial lesions occurred the day after each VIT injections. Nevertheless, after few months of VIT both pruritus and wheals improved gradually, and after ten months urticaria suddenly remitted and antihistamines were stopped. At present CU symptoms have not recurred and the patient continues VIT (almost 5 years so far).

\section{DISCUSSION}

The hypothesis behind urticaria progression can be explained from several perspectives. First, vespid sting and urticaria onset could be coincidental and CU remitted spontaneously with VIT exerting no effect on its course. In fact, in most cases, CU is a self-limiting disorder, persisting for 2-5 years. ${ }^{2}$ Moreover, Hymenoptera sting causes acute urticaria and in the literature no report of CU after Hymenoptera sting can be found.

Alternatively, CU progression, from onset to remission, might be associated with some specific effects of venom allergens, from sting to VIT. Supporting this hypothesis, we observed a clear-cut association between exposure to venom allergens and urticaria symptoms. In fact, type I allergy is a rare cause of CU, but should be considered in patients with intermittent symptoms. ${ }^{3}$ Examples of CU treated with allergen immunotherapy (AIT) can be found in literature. Immunotherapy with sweat extract has been reported to be successful in the treatment of cholinergic urticaria, presumably due to the induction of tolerance to endogenous allergens. ${ }^{4}$ Sublingual immunotherapy with latex extract showed efficacy in patients with latex-induced urticaria. case of seasonal urticaria due to grass pollinosis was treated successfully with desensitization. ${ }^{6}$ House dust mite allergy has been hypothesized as a pathogenic factor in CU, and some patients have benefited from AIT. 
study reported an improvement in CU symptoms using AIT with Giardia's antigen preparation. ${ }^{8}$

Moreover, besides its specific effects, AIT exerts some non-specific effects. AIT changes basophil and mast cell homeostasis and reduces the skin sensitivity not only to specific allergens, but also to histamine and non-specific mast cell stimuli. Immunologic changes induced during the course of AIT include reduced mast cell and basophil activity and degranulation. After a few months, AIT produces a decrease in tissue mast cell, both connective and mucosal, in innate type 2 lymphocytes and eosinophil counts as well as in the release of their mediators. ${ }^{9,10}$ As a matter of fact, reduced immune cells reactivity could contribute significantly to the improvement of urticaria symptoms, even if they have not been related to venom allergens. Therefore, in our patient VIT might have played a positive role, increasing the sensitivity threshold to non-specific stimuli.

\section{CONFLICT OF INTEREST}

The authors certify that there is no conflict interest with any financial organization regarding the material discussed in the manuscript.

\section{AUTHOR CONTRIBUTIONS}

VP and FR provided the patient data regarding the allergic disease; MD and VP wrote the manuscript; RA and GS made contributions to interpretation of data. All authors critically read and approved the final manuscript.

\section{ETHICS STATEMENT}

All procedures were in accordance with the ethical standards of the responsible committee on human experimentation (institutional and national) and with the Helsinki Declaration of 1975, as revised in 2013. Informed written consent was obtained from the patient.

\section{DATA AVAILABILITY STATEMENT}

The data that support the findings of this study are available from the corresponding author upon reasonable request.

\section{ORCID}

Marco Dubini iD http://orcid.org/0000-0003-1760-783X

\section{REFERENCES}

1. Zuberbier T, Aberer W, Asero R, Abdul Latiff AH, Baker D, Ballmer-Weber B, et al. The EAA$\mathrm{CI} / \mathrm{GA}^{2} \mathrm{LEN} / \mathrm{EDF} / \mathrm{WAO}$ guideline for the definition, classification, diagnosis and management of urticaria. Allergy. 2018 Jul;73(7):1393-414.

2. Vestergaard C, Toubi E, Maurer M, Triggiani M, Ballmer-Weber B, Marsland A, et al. Treatment of chronic spontaneous urticaria with an inadequate response to H1-antihistamines: an expert opinion. Eur J Dermatol. 2017;27(1):10-9.

3. Shi CR, Li YP, Luo YJ, Shi CB, Yan X, Yang KH,et al. IgE-mediated allergy: a rare cause of chronic spontaneous urticarial with allergen-specific immunotherapy as treatment option - a systematic review with meta-analysis from China. J Eur Acad Dermatol Venereol. 2012 May;26(5):533-44.

4. Kozaru T, Fukunaga A, Taguchi K, Ogura K, Nagano T, Oka M, et al. Rapid desensitization with autologous sweat in cholinergic urticaria. Allergol Int. 2011 Sep;60(3):277-81.

5. Nettis E, Di Leo E, Calogiuri G, Milani M, Delle Donne P, Ferrannini A, et al. The safety of a novel sublingual rush induction phase for latex desensitization. Curr Med Res Opin. 2010;26:1855-59.

6. August PJ, O'Driscoll J. Urticaria successfully treated by desensitization with grass pollen extract. Br J Dermatol. 1989 Mar;120(3):409-10.

7. Kasperska-Zajac A, Brzoza Z. Remission of chronic urticaria in the course of house dust mite immunotherapy - mere coincidence or something more to it? Vaccine. 2009;27:7240-1.

8. Abdo Rodriguez A, Espino Anton P, Zaldivar Estevez JC. Immunotherapy using Giardia lamblia extract in recurrent chronic sensitized urticaria [in Spanish]. Rev Alerg. 1991;38:113-6. 
9. Akdis M, Akdis CA. Mechanisms of allergen-specific immunotherapy: multiple suppressor factors at work in immune tolerance to allergens. J Allergy Clin Immunol. 2014 Mar;133(3):621-31.

10. Sahiner UM, Durham SR. Hymenoptera Venom Allergy: How Does Venom Immunotherapy Prevent Anaphylaxis From Bee and Wasp Stings? Front Immunol. 2019 Aug 21;10:1959. 